\title{
CD44 as a Biomarker in the Early Detection of Breast Cancer
}

\author{
Ali Tafti ${ }^{1,}$, Minoo Shahani ${ }^{2}$, Mohammad Amin Mahmanzar ${ }^{1}$, Binazir \\ Khanabadi ${ }^{1}$, Jafar Shakeri ${ }^{3}$ \\ ${ }^{1}$ Department of Cellular and Molecular Biology, Tehran Medical Sciences Branch, \\ Islamic Azad University \\ ${ }^{2}$ Cancer Research Center, Shahid Beheshti University of Medical Sciences, Tehran, \\ Iran \\ ${ }^{3}$ Oncosurgeon Cancer Research Center, Shahid Beheshti University of Medical \\ Sciences, Tehran, Iran \\ *Corresponding Author: Ali Tafti, Department of Cellular and Molecular Biology, \\ Tehran Medical Sciences Branch, Islamic Azad University.E-mail: tafti1375@gmail. \\ com
}

DOI: $10.21859 /$ mci-supp-40

\section{Keywords:}

Breast Cancer

Cancer Stem Cell

Biomarker

Early Detection

\begin{abstract}
Introduction: Due to the high prevalence of low-risk breast cancer (BC) and the side effects affecting families in Iran, related researches are necessary. BC is one of the main leading causes of death in women, causing 40,000 to 50,000 deaths per year. Therefore, the recognition of prognostic factors, biomarkers and preventive factors can be useful for selecting appropriate therapies and clinical decisions.

Materials and Methods: According to Keywords: Breast Cancer, Biomarkers, Early detection and cancer Stem cell that utilized the valid databases, such as: Scopus, NCBI and Elsevier. The papers collected and then was ranking based on appropriate criteria.

Results: In this study, according to key words in the field of Biomarkers involved in Breast Cancer 65 articles were selected. After completing the final analysis, 15 studies from other research more relevant to their subject, were assessed.

Conclusions: The heterogeneous and difficult nature of the $\mathrm{BC}$ is becoming a leading condition to define, in accordance with its growing incidence and poor. According to of Results, identify CD44 as a biomarker that is important in the early detection of Breast Cancer.
\end{abstract}

